\title{
A SIMPLIFIED APPROACH FOR MODELLING AIRBORNE NANOPARTICULES TRANSPORT AND DIFFUSION
}

\author{
FRANÇOIS MORENCY \& STÉPHANE HALLÉ \\ Département de génie mécanique, École de technologie supérieure, Canada.
}

\begin{abstract}
A simplified approach is proposed and used to study the $\mathrm{TiO}_{2}$ nanoparticle transport and diffusion in an exposure chamber. This exposure chamber is used to assess lung toxicity in rats resulting from the inhalation of airborne nanoparticles. The simplified approach uses computational fluid dynamics (CFD) commercial software. The mathematical model for airflow is based on the three-dimensional Reynoldsaveraged Navier-Stokes equations with turbulence modeling. The mathematical model for airborne nanoparticles transport is based on assumptions such that their motions are similar to those of a singlesized diameter distribution of a passive contaminant. This model is valid as long as the nanoparticle concentration is low and the particle diameter is small enough that settling is negligible, which is the case for the exposure chamber studied. With this model, the diffusion coefficient is a property that plays a significant role in the transport of airborne nanoparticles. The particle diffusion coefficient can be expressed in terms of a friction coefficient, and three possible relationships to model particle diffusion are presented. Their influences on the friction and diffusion coefficients are considered for the particular case of $\mathrm{TiO}_{2}$ nanoparticles. Although all the models studied here predict a decrease in the value of the diffusion coefficient with increasing particle diameter, some significant variations can be observed between the models. A specific diffusion model is selected and then used with the simplified approach. The simplified approach is first validated against available correlations for particle deposition on walls. Correlation for deposition loss rate in the case of a room agrees with numerical prediction for particle diameter between 10 and $200 \mathrm{~nm}$. Particle mass concentration distribution inside the exposure chamber is also studied. The computed concentration distribution is quite uniform inside the exposure chamber and corresponds to single point measurements.
\end{abstract}

Keywords: CFD, diffusion, exposure chamber, nanoparticles, particle deposition, passive contaminant, purge time, titanium oxide.

\section{INTRODUCTION}

The evaluation of the potential hazards for human health associated with inhalation or other forms of contact with nanoparticles (NPs) is a question of great interest in the scientific community. Indeed, the phenomenal emergence of various types of nanotechnologies has led many governmental agencies to present discussion papers on the safe use of nanometric-scale particles, herein called NPs. These international reports are unanimous in supporting proactive measures to ensure the safety of workers exposed to NPs, as reports from Aitken et al. [1] and Ostiguy et al. [2] emphasize.

One of the basic elements for risk assessment evaluation in a work environment consists of an adequate characterization of the degrees of exposure. In the case of airborne NPs, numerical simulations could be used advantageously as a tool to safely predict exposure levels. These simulations would require the development of appropriate models. It is expected that, once validated, these models will make it possible to predict the behavior of airborne NPs in a workplace environment, to evaluate the effective exposure level to these NPs, and to safely assess the associated risks. These results will allow the design of efficient ventilation and/or filtering systems that could also make it possible to contain and to recover from an accidental release of undesirable NPs. To reach these objectives, the numerical models should be able to represent the dominant particles' transport and dispersion mechanisms. 
In that perspective of developing reliable models to simulate the transport and dispersion of airborne NPs, the objective of this paper is to present numerical results for a simple model of NP dispersion in an animal exposure chamber. This exposure chamber will be used in further studies to assess lung toxicity in rats resulting from the inhalation exposure to airborne titanium dioxide $\left(\mathrm{TiO}_{2}\right) \mathrm{NPs}$. The NP concentration inside the exposure chamber is low enough that the agglomeration phenomenon is negligible. Also, the NPs are small enough that gravitational settling is negligible.

More specifically, the air flow simulation results are presented in the exposure chamber for which the NPs are assumed to behave as a passive contaminant. With this assumption, the passive contaminant (the NPs) is transported by convection and diffusion only. Thus, the diffusion coefficient is a key parameter in the approach. First, mathematical model for evaluating NP diffusion coefficients are presented, together with empirical relations to estimate the diffusion loss of particles at walls. Then, the mathematical model used for the numerical simulation of airborne NP transport will be detailed. The proposed mathematical model is validated against empirical relations for particle deposition inside small 2D and 3D rooms. Finally, a description of the exposure chamber is provided, prior to the presentation of the numerical results and their comparison with experimental measurements made in the chamber.

\section{NANOPARTICLE DIFFUSION COEFFICIENT}

Classical theory relates the Brownian motion of small particles to the molecular motion of gas molecules (see Hinds [3]). The Stokes-Einstein expression for the diffusion coefficient is

$$
D=\frac{K T}{f}
$$

where $K$ is the Boltzmann constant and $T$ stands for the absolute temperature. The friction coefficient $f$ is dependent upon the particle size and the fluid physical properties.

We will consider here three possible expressions to evaluate this NP friction coefficient. These equations all include the Knudsen number, Kn, defined here as twice the ratio of the mean free path of the gas molecules, $l_{p}$, to the particle aerodynamic diameter $d_{p}$, such that

$$
\mathrm{Kn}=\frac{2 l_{p}}{d_{p}} .
$$

For a $\mathrm{Kn}<1$, the Stokes law may be applied. If the particle is considered as rigid and spherical, the friction coefficient can be written as

$$
f=3 \pi \mu d_{p} .
$$

According to Friedlander [4], for a $\mathrm{Kn}>1$, the friction coefficient can then be determined according to the Enskog kinetic theory, so that

$$
f=\frac{2}{3} d_{p}^{2} \rho\left(\frac{2 \pi k T}{m}\right)^{1 / 2}\left(1+\frac{\pi \alpha}{8}\right)
$$

where $\rho$ is the gas 2 density, $m$ is the molecular mass of the gas molecules, and the accommodation coefficient $\alpha$ is a constant parameter usually assumed to have a numerical value of 0.9 . 
A generalization of the above relations can be made to cover a more global range of $\mathrm{Kn}$ numbers by rewriting the Stokes law, eqn (3), with a correction factor $C$ such that

$$
f=\frac{3 \pi \mu d_{p}}{C} .
$$

The effects of the Kn are then included in the correction factor, the value of which can in turn be determined by more than one relation. Friedlander [4] and Rudyak et al. [5] suggest the following expression

$$
C=1+\mathrm{Kn}\left(1.257+0.400 \exp \frac{-1.1}{\mathrm{Kn}}\right)
$$

derived from experimental data for oil droplets in air. Hinds [3] proposes a slightly different relation in the form of

$$
C=1+\frac{\mathrm{Kn}}{2}\left(2.34+1.05 \exp \frac{-0.78}{\mathrm{Kn}}\right)
$$

Finally, Gussman [6] suggests a relation applicable for different types of gas which reduces to

$$
C=(1+0.615 \mathrm{Kn})^{-1}+1.615 \mathrm{Kn}
$$

for air.

Results for the different models are compared in detail by Morency et al. [7], together with temperature effects on the diffusion coefficients. Figure 1 compares the coefficient $D$ predicted by (i) Friedlander's eqn (7), (ii) Gussman's eqn (8), (iii) the kinetic theory of eqn (4), and (iv) the Stokes law in eqn (3). The numerical values shown were obtained for airborne $\mathrm{TiO}_{2}$ particles in air at standard ambient pressure, $1 \mathrm{~atm}$, and temperature, $20^{\circ} \mathrm{C}$. As can be

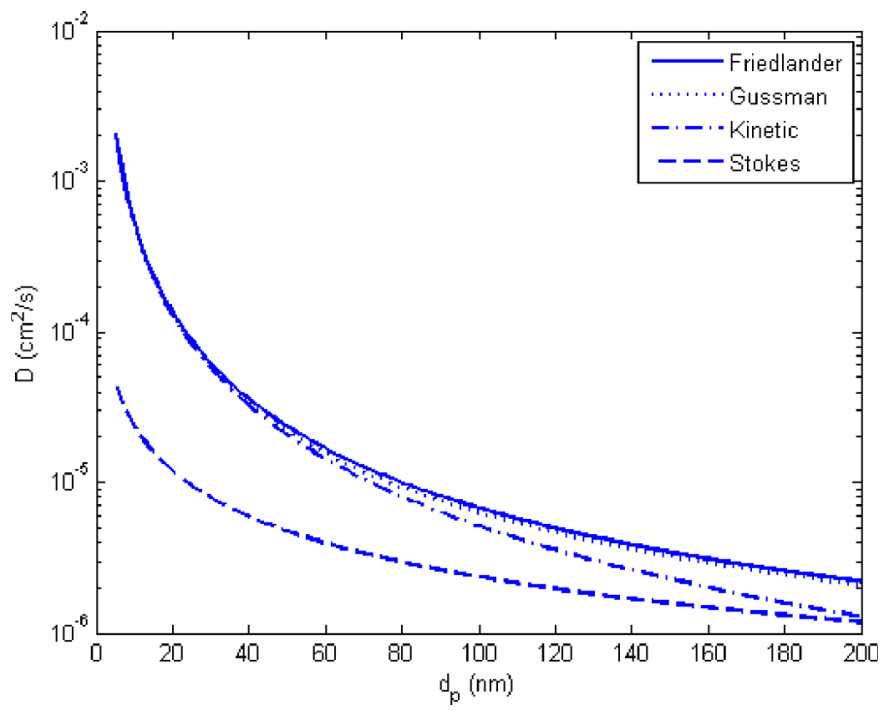

Figure 1: Diffusion coefficient for $\mathrm{TiO}_{2}$ particle in air at atmospheric pressure. 
seen in Fig. 1, the diffusion coefficients decrease as particle size increases from 10 to $200 \mathrm{~nm}$. As expected, the Stokes law under-predicts the values of $D$. For particle diameters greater than about $40 \mathrm{~nm}$, the kinetic theory gives different results from those obtained from Friedlander's and Gussman's equations, which do, however, agree quite well with each other. Hinds' equation results are not shown, as they are almost identical to those of Friedlander.

Morency et al. [7] showed that the coefficient $D$ is proportional to the temperature of the medium and inversely proportional to the square of the particle aerodynamic diameter $d_{p}$. Despite this dependence, the diffusion coefficient of a NP as small as $1 \mathrm{~nm}$ in diameter will be 20 times smaller than the equivalent coefficient for air (molecules). The diffusion of the NPs in air is therefore unlikely to change their trajectory significantly compared to the main flow streamlines, as stated by Maynard [8]. This 'passive scalar' behavior implies that the NPs can be transported directly by the convective movement of a fluid. However, the diffusion has a determining influence on the 'particle-particle' collision probability and on the 'particle-wall' collision probability. Relations for coagulation rate predictions are presented by Hinds [3], but in the present study, the particle number concentrations are low enough, and, considering the time scale involved in our simulations, the 'particle-particle' collision probability is negligible.

\subsection{Diffusional loss of nanoparticles}

Empirical models for particle deposition on surfaces from turbulent flow in enclosure are reviewed by Lai [9]. These models aim to determine the particle decay rate loss coefficient, $\beta$, for airborne particles into a well-mixed enclosure. Under these conditions, the particle concentration would decay with time as

$$
C(t)=C_{0}(\exp (-\beta t)
$$

where $t$ is the elapsed time and $C_{0}$ is the initial concentration. The particle decay coefficient includes both diffusion loss to the wall and gravitational settling. Several models to predict the particle decay coefficient have been proposed in the literature, all based on the assumption that particle concentrations are constant and uniform outside the concentration boundary layer near a wall's enclosure.

Initial work by Corner and Pendlebury [10] relates the particle decay coefficient to sedimentation velocity and Brownian particle diffusivity by

$$
\beta=\frac{v_{s}}{L_{1}}+\frac{S D}{V \sigma}
$$

where $v_{s}$ is the particle sedimentation velocity, $L_{1}$ is the vessel height, $V$ and $S$ are the vessel volume and surface area, $D$ is the Brownian particle diffusivity, and $\sigma$ is the boundary layer diffusion thickness. The boundary layer diffusion thickness depends mainly on the vessel shape, particle size, and airflow. It is a hypothetical quantity whose value is derived from its comparison with experimental results for a given geometry.

Crump and Seinfeld [11] proposed models where vessel shape and convective mixing are accounted for. For example, the decay coefficient in a rectangular box is

$$
\beta=\frac{2 v_{s}}{X_{1}}\left(\frac{1}{L_{2}}+\frac{1}{L_{3}}\right)+\frac{v_{s}}{L_{1}} \operatorname{coth}\left(\frac{X_{1}}{2}\right)
$$


where,

$$
X_{1}=\frac{\pi v_{s}}{n \sin \left(\frac{\pi}{n}\right) \sqrt[n]{k_{e} D^{n-1}}}
$$

$L_{1}, L_{2}$, and $L_{3}$ represent, respectively, the height, length, and width of the rectangular box. The variables $k_{e}$ and $n$ are the coefficient and exponent of the eddy diffusivity coefficient $k_{e}$, defined by

$$
D_{e}=k_{e} y^{n}
$$

where $y$ is the normal distance from the wall. Chen et al. [12] investigated the appropriate values for parameters $k_{e}$ and $n$. They found values between 2 and 3 for $n$ in the literature and suggested using a value close to 2.6. The parameter $k_{e}$ characterizes the intensity of turbulent mixing within the chamber and is dependent upon the flow conditions inside the chamber.

In the limit of ultrafine particles or NPs, the sedimentation velocity becomes negligible. Equations (11) and (12) can then be replaced by the following eqn. [13].

$$
\beta=\frac{S}{V} \frac{n}{\pi} \sin \left(\frac{\pi}{n}\right) \sqrt[n]{k_{e} D^{n-1}} .
$$

Lai and Nazaroff [14] proposed a model that accounts for the effect of Brownian and turbulent diffusion and gravitational settling based on friction velocity, defined as

$$
u^{*}=\sqrt{\frac{\tau_{w}}{\rho}} .
$$

This new model predicts results similar to Crump and Seinfeld's model if proper values of parameters $n$ and $k_{e}$ are selected. To have agreement between the two models, the suggested value for $n$ is 2.94, compared to the value of 2.6 suggested by Chen et al. [12], based on a review of the experimental results. In the present study, eqns (10) and (11) are used for numerical calculation verifications.

\section{MATHEMATICAL MODEL}

The flow inside various geometries is first computed by solving the Reynolds-averaged incompressible Navier-Stokes equations. Then, the NP concentration field is estimated using a passive contaminant approach based on Eulerian Method.

\subsection{Governing equations of turbulent airflow}

Airflow within the exposure chamber is modeled by 3D Reynolds-averaged NavierStokes (RANS) equations using the eddy viscosity $\left(v_{\mathrm{t}}\right)$ assumption. In Cartesian coordinates, the mass conservation equation, eqn (15), and the momentum conservation equation, eqn (16), are

$$
\frac{\partial U_{i}}{\partial x_{i}}=0
$$




$$
U_{j} \frac{\partial U_{i}}{\partial x_{j}}=-\frac{1}{\rho} \frac{\partial P}{\partial x_{i}}+\left[\left(v+v_{t}\right)\left(\frac{\partial U_{i}}{\partial x_{j}}+\frac{\partial U_{j}}{\partial x_{i}}\right)\right]
$$

where $U_{i}$ are the velocity components, $x_{i}$ are rectilinear orthogonal coordinates, $P$ is the pressure, $\rho$ the density, and $v$ and $v_{t}$ are the dynamic viscosity of air and the turbulent viscosity, respectively.

Turbulent effects are modeled by the $k-\omega$ SST turbulence model. In this model, the eddy viscosity is a function of the turbulent kinetic energy $(k)$ and the specific dissipation rate $(\omega)$. Both variables can be determined by solving two additional transport equations. More details on the $k-\omega$ SST turbulence model are available in Menter [15]. ANSYS Fluent [16] software uses an enhanced wall treatment method for this SST turbulence model that combines a twolayer zonal model when the mesh is fine enough to resolve the laminar sublayer and enhanced wall functions when the mesh is coarser [17].

\subsection{Governing equations for the NPs transport}

Airborne NPs are subjected to numerous physical phenomena that shape their size distribution in space and time. For example, coagulation leads to a reduction of the total number of particles, to an increase in the average particle diameter, and to an increase in the number of molecules in the particles. Particle growth occurs by gas-to-particle conversion. Sedimentation resulting from the gravity field also occurs, with the particle settling velocity dependent upon particle size.

However, under a certain hypotheses, NPs size and number distributions can be considered constant in space and time: (i) for low NP concentration, no significant coagulation or agglomeration of NPs will occur, as suggested by Hinds [3], and thus aerosol distribution is not altered; (ii) if there is no gas-to-particle conversion; and (iii) when sedimentation or settling of NPs is negligible in the global dispersion process, as current studies by Lechner [18] indicate. Moreover, because of their low concentration, NPs have no effects on the air flow and behave as a passive contaminant. This approximation is widely used for clean room design, for example, by $\mathrm{Hu}$ et al. [19] and Zhao and Wu [20]. Finally, if the NP distribution is well represented by a single mean aerodynamic diameter, the particle concentration is governed by the following Eulerian model

$$
\frac{\partial\left(\rho Z_{A} U_{i}\right)}{\partial x_{i}}=\frac{\partial}{\partial x_{i}}\left[\left(\rho D+\frac{\rho v_{t}}{S c_{t}}\right)\left(\frac{\partial Z_{A}}{\partial x_{i}}\right)\right]
$$

In eqn (17), $Z_{A}$ is the ratio of the particles' mass fraction to the total mass, $D$ is the NPs' Brownian diffusion coefficient, and the turbulent Schmidt number $\left(S c_{t}\right)$ represents the ratio of the air eddy viscosity to the eddy mass diffusivity. The turbulence in the exposure chamber will generate diffusion effects which are orders of magnitude higher than Brownian diffusion. These turbulent diffusion effects are characterized by the turbulent Schmidt number which is usually close to unity; therefore $S c_{t}$ was fixed to 0.9 . However, the intensity of turbulence becomes negligible near solid walls and the Brownian diffusion is the dominant mechanism in that region.

A Eulerian approach, similar to the one proposed here, has been used by other researchers, such as Zhang et al.'s [21] study of NP deposition in the human tracheobronchial region and 
Kumar et al.'s [22] study of NP concentration in an urban street canyon. Lai and Chen [23] proposed an Eulerian model that includes gravitational settling to simulate particle dispersion in a chamber. They simply added the particle settling velocity $v_{\mathrm{s}}$ to the air velocity $U$ on the left of eqn (17). As we will see in the following section, for an aerodynamic diameter of $<100 \mathrm{~nm}$, a particle's settling velocity is negligible compared to the air velocity. Thus, eqn (17) is a valid approximation of Lai and Chen's model for NP transport.

\subsection{Numerical method}

In the present study, the set of coupled equations, eqns (15), (16), and (17), are solved by a finite volume method using the commercial software ANSYS Fluent version 12.1. The discretization method is based on the SIMPLE algorithm and a first-order upwind scheme. NPs and air are mixed at a molecular level (multispecies formulation). The flow is considered isothermal, turbulent, and incompressible.

The inlet is defined as an opening with a uniform velocity profile and a constant mass fraction. At the outlet, mass conservation boundary conditions for all velocity components and a zero-gradient mass concentration condition for the passive contaminant are applied. Diffusional losses are considered by imposing a zero NP mass fraction at the walls. This boundary condition assumes that NPs are trapped at the walls and neglects their rebound. The initial conditions are: the fluid is assumed to be perfectly still $\left(U_{i}=0\right)$ and the NP mass fraction is zero in the computational domain.

The convergence criteria for the resolved velocities and pressure are $10^{-6}$ and $10^{-5}$, respectively. Particle mass concentration $\left(Z_{A}\right)$ convergence criterion is $10^{-6}$ and $10^{-3}$ for the turbulent quantities ( $k$ and $\omega$, respectively). Under-relaxation parameters were set to 0.3 for pressure, 0.7 for momentum equations, and 1 for the mass transport equation.

\section{RESULTS}

Two test case results are presented here prior to presenting the calculation results for the inhalation chamber. Due to a lack of experimental data, the particle concentration field inside a chamber cannot be validated. Current measurement apparatus do not enable instantaneous evaluation of particle concentration at several points simultaneously. However, it is possible to compare the decay coefficient obtained numerically against empirical relations developed to predict the decay coefficient. Given that the overall decay coefficient is dependent on the concentration distribution near the chamber wall, it provides a qualitative validation of the simplified model. In a previous work by Morency and Hallé [24], computational results were validated against correlations for transport efficiency in a turbulent pipe flow. Their results agreed with that of the correlation predicted value within $6 \%$. In this section, decay coefficients for a 2D square chamber are presented, followed by decay coefficients obtained in a $3 \mathrm{D}$ cubic chamber. The results for the inhalation chamber are then presented and discussed.

\subsection{Particle extraction from a 2D square chamber}

A $2 \mathrm{D}$ chamber is a particular case of a 3D chamber in which the chamber depth is infinite. A square chamber of $100 \times 100 \mathrm{~cm}$ is illustrated schematically in Fig. 2, part (a). Its inlet and outlet are of the same size, $1 \mathrm{~cm}$. The inlet is at the top left corner. Two constant inlet velocities are tested, $1 \mathrm{~m} / \mathrm{s}$ and $0.5 \mathrm{~m} / \mathrm{s}$, corresponding to air exchange rates of $0.01 \mathrm{~m}^{3} / \mathrm{s}\left(36 \mathrm{~h}^{-1}\right)$ and $0.005 \mathrm{~m}^{3} / \mathrm{s}\left(18 \mathrm{~h}^{-1}\right)$, respectively. Air temperature is set as $25^{\circ} \mathrm{C}$, which corresponds to 
Reynolds numbers based on inlet sizes of 640 and 320 above the transition Reynolds number for plane jets [25]. The entrance turbulence level is defined by a $5 \%$ turbulence intensity and a turbulent viscosity ratio, $v_{t} / v$, equal to 10 . The velocity vector is normal to the boundary. Tests with various velocity vector directions at the inlet show that decay coefficients are insensitive to inlet configuration. Decay coefficients are also not very sensitive to inlet geometry. For example, for the same flow rate, numerical calculations with a 10-times wider inlet predict virtually the same decay coefficients.

The square chamber is discretized in finite volume of hexahedral shape. The results shown in Fig. 2(b) were obtained with a $480 \times 320$ node mesh. The mesh is non-uniform with node concentrations near the walls, inlet, and exit. The distance between first node and the wall is $0.1 \mathrm{~mm}$, and a geometric progression with a ratio of 1.2 was used. For the $1 \mathrm{~m} / \mathrm{s}$ velocity, the maximum $\mathrm{y}^{+}$value was around 1.29 with an average value of 0.72 , and the average friction velocity was $2.2 \mathrm{~cm} / \mathrm{s}$. For the $0.5 \mathrm{~m} / \mathrm{s}$ velocity, the maximum $\mathrm{y}^{+}$value was around 0.79 with an average value of 0.039 , and the average friction velocity was $1.2 \mathrm{~cm} / \mathrm{s}$. Thus, the velocity boundary layer was well resolved with the proposed mesh. Two others meshes were also tested: one coarser $(240 \times 160$ nodes $)$ and one finer $(960 \times 640)$. Table 1 compares the decay coefficients for three different particle sizes and a $0.5 \mathrm{~m} / \mathrm{s}$ inlet velocity. For a $10 \mathrm{~nm}$ particle, there is $<1 \%$ of difference between the medium grid and the fine grid. As the particle gets

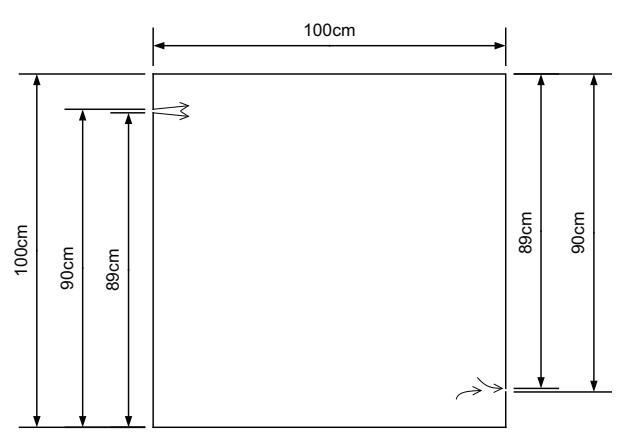

(a)

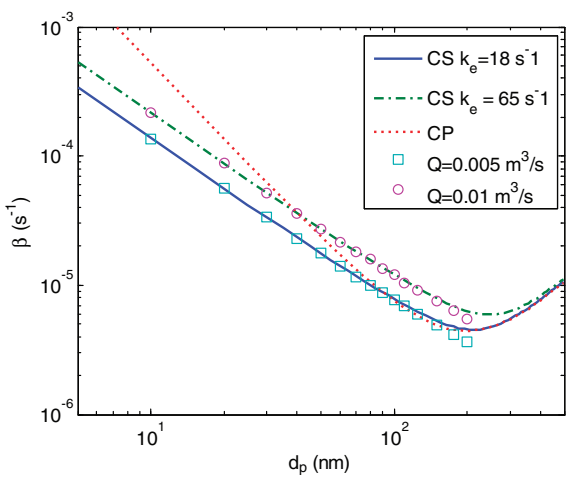

(b)

Figure 2: A 2D square chamber geometry (a) and corresponding decay coefficients $(n=2.94$, $\sigma=0.4 \mathrm{~mm})(\mathrm{b})$.

Table 1: Variation of decay coefficients with mesh size (2D case).

\begin{tabular}{lccc}
\hline \multicolumn{3}{c}{$\beta[1 / \mathrm{s}]$} \\
\cline { 2 - 4 }$d_{p}[\mathrm{~nm}]$ & $240 \times 160$ nodes & $480 \times 320$ nodes & $960 \times 640$ node \\
\hline 10 & $1.401 \mathrm{e}-4$ & $1.364 \mathrm{e}-4$ & $1.359 \mathrm{e}-4$ \\
100 & $8.592 \mathrm{e}-6$ & $7.666 \mathrm{e}-6$ & $7.299 \mathrm{e}-6$ \\
200 & $4.022 \mathrm{e}-6$ & $3.628 \mathrm{e}-6$ & $3.368 \mathrm{e}-6$ \\
\hline
\end{tabular}


larger, the diffusion coefficient gets smaller and the diffusive boundary layer requires more nodes close to the wall, even if the velocity boundary layer is well-resolved. Consequently, for the larger particle diameters, the solution still changes by about $7 \%$ between the medium grid and the fine grid. However, the proposed model is not valid for the larger diameter, as gravity effects are neglected.

In Fig. 2, part (b), the decay coefficients are plotted against particle diameter, with a log$\log$ scale. The numerical results, square and round dots, are compared with prediction from eqn (10), representing the Corner and Pendlebury (CP) model, and eqn (11), called CS, for the Crump and Seinfeld model. The decay coefficients are obtained from mass flow average concentration at the inlet $C_{\text {in }}$ and the exit, $C_{\text {out }}$, with the following equation [26]:

$$
\beta=\frac{Q\left(C_{\text {in }}-C_{\text {out }}\right)}{V C_{\text {out }}} .
$$

Because gravity effects are neglected, decay coefficients continuously decrease as particle diameters increase. In reality, as the empirical models show, decay coefficients should start to increase for particle diameters $>200 \mathrm{~nm}$. Thus, the proposed NP transport equation is valid until diameters reach around $100 \mathrm{~nm}$.

For diameters at/or $100 \mathrm{~nm}$, the decay coefficients show a log-linear dependence with the particle diffusion coefficient. The slope of the numerical results is 0.66 for both $Q=0.05 \mathrm{~m}^{3} / \mathrm{s}$ and $\mathrm{Q}=0.001 \mathrm{~m}^{3} / \mathrm{s}$.

The slope of the empirical models can be obtained, if we neglect the sedimentation velocity. Equation (10) then becomes

$$
\beta=\frac{S D}{V \sigma}
$$

and if we take the logarithm on both sides

$$
\log \beta=\log \left(\frac{S}{V \sigma}\right)+\log D
$$

then the slope of the CP model is 1 .

Similarly, eqn (14) valid for particle diameters under $100 \mathrm{~nm}$ becomes

$$
\log \beta=\log \left(\frac{S}{V} \frac{n}{\pi} \sin \frac{\pi}{n} \sqrt[n]{k_{e}}\right)+\frac{n+1}{n} \log D
$$

The slope of the CS model is $(n-1 / n)$. For $(\mathrm{n}=2.94)$, the slope is 0.66 . This value corresponds to the slope of our numerical results. The value of 2.94 was also suggested by Lai and Nazaroff [14].

\subsection{Particle extraction from a 3D square chamber}

Figure 3(a) shows the 3D cubic chamber. Air and NPs are blown into the $1 \mathrm{~m}^{3}$ chamber via a $2.5 \times 10^{-3} \mathrm{~m}^{2}$ inlet located at the top left corner. The mixture is then extracted at the outlet located at the bottom right corner. This specific supply and return grill arrangement is designed to create perfect mixing condition in the computational domain. As shown in Fig. 3(b), the air flow from the supply vent hits the opposite wall and is distributed uniformly 


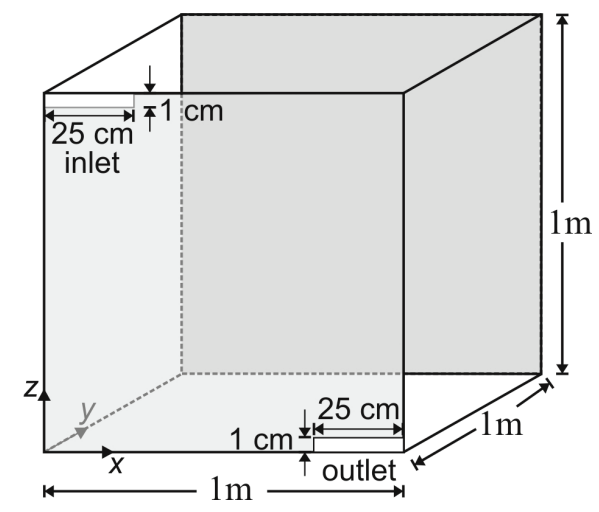

(a)

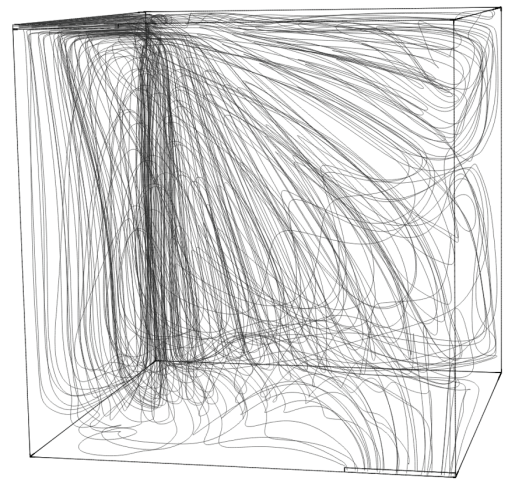

(b)

Figure 3: A 3D rectangular chamber geometry (a) and airflow streamlines (b).

in the chamber. Simulations were performed for a constant inlet velocity of $1.5 \mathrm{~m} / \mathrm{s}$, corresponding to air exchange rates of $13.5 \mathrm{~h}^{-1}$. The corresponding Reynolds number based on the inlet hydraulic diameter is 1859 . As in the $2 \mathrm{D}$ case, air temperature is set as $25^{\circ} \mathrm{C}$, and a $5 \%$ turbulence intensity and a viscosity ratio, $v_{t} / v$, equal to 10 , defines the turbulence level at the entrance. Spatial discretization is accomplished by subdividing the chamber into parallelepiped elements. Three grids were studied from coarsest (with 1,688,208 elements) to finest (with 3,821,304 elements). To capture the velocity and concentration gradients, the mesh density is higher near the walls and at the air inlet/outlet. This refinement led to maximum $\mathrm{y}^{+}$values of the order of 7,4 , and 2 for the coarse, medium, and fine meshes, respectively.

Decay coefficients were computed for the three meshes. Numerical results obtained for the finer mesh using eqn (18) are presented in Fig. 4. These results, plotted on a log-log scale, are compared with those of the CP model, eqn (19), with $\sigma=0.9 \mathrm{~mm}$, and of the modified CS model, eqn (14), with parameters $k_{e}$ and $n$ fixed to $22 \mathrm{~s}^{-1}$ and $2.9 \mathrm{~s}^{-1}$, respectively. Numerical predictions agree quite well with both models, except that, unlike the 2D case for which the results closely follow the CS model, decay coefficients for the cubic chamber do not follow a particular model. The slope obtained from our results is 0.86 , a numerical prediction slightly higher than the slope obtained from the CS model, with $n=2.9$, and lower than the value predicted from the CP model.

Decay coefficients obtained for $10 \mathrm{~nm}$ and $100 \mathrm{~nm}$ particles are compared in Table 2 for the three mesh densities. For both diameters, the difference between the medium and fine mesh is about $1 \%$. However, the $\beta$ obtained from the coarse mesh differ significantly from the fine mesh, with deviations as large as $34 \%$. These results indicate that a very fine grid near the wall is required to obtain a grid-independent result for the decay coefficient.

As an additional means to assess the influence of mesh density on the numerical solution, the volumetric flow rates through a rectangular surface of $0.005 \mathrm{~m}^{2}$ delimited by the coordinates $(0.0,0.5,0.98)$ and $(0.25,0.5,1.0)$ are compared in Table 3 . This vertical plane is located downstream in front of the inlet. The flow rates for the medium and fine mesh are almost identical, with a difference on the order of $0.2 \%$. 


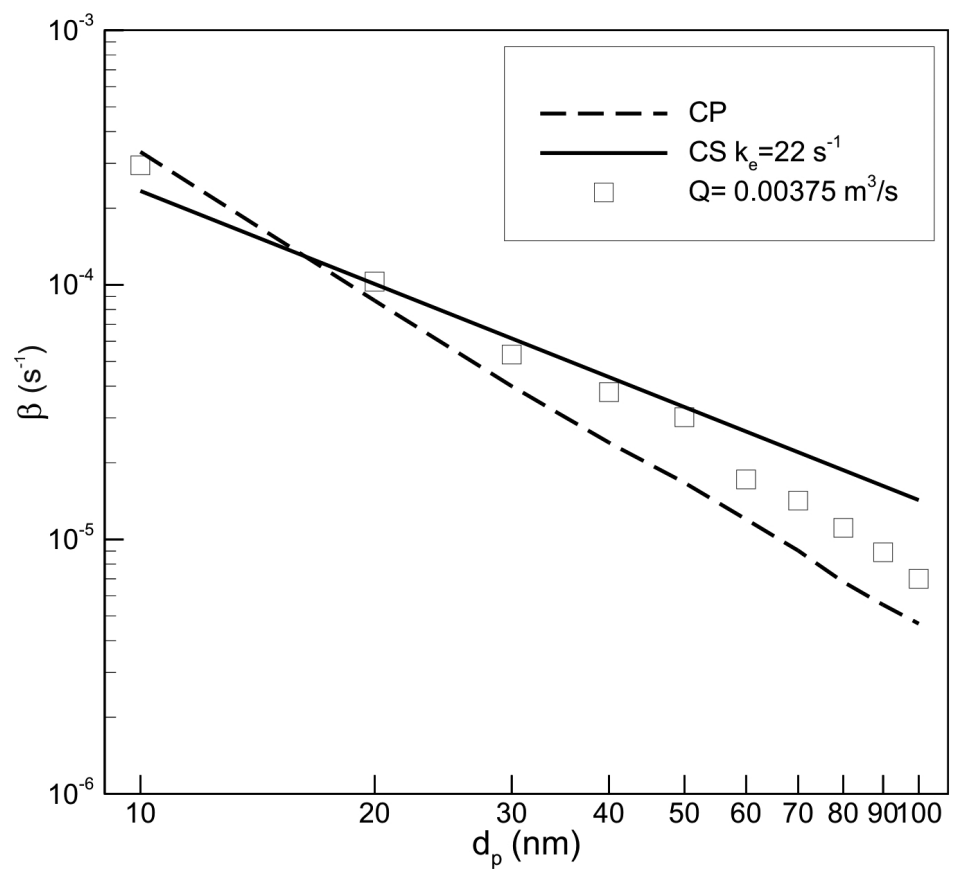

Figure 4: Decay coefficient for the 3D case $(n=2.90, \sigma=0.9 \mathrm{~mm})$.

Table 2: Variation of decay coefficient with mesh size (3D case).

\begin{tabular}{lccc}
\hline & \multicolumn{3}{c}{$\beta[1 / \mathrm{s}]$} \\
\cline { 2 - 4 }$d_{p}[\mathrm{~nm}]$ & $1,688,208$ elements & $3,593,048$ elements & $3,821,304$ elements \\
\hline 10 & $2.282 \mathrm{e}-4$ & $2.920 \mathrm{e}-4$ & $2.945 \mathrm{e}-4$ \\
100 & $9.432 \mathrm{e}-6$ & $7.103 \mathrm{e}-6$ & $7.021 \mathrm{e}-6$ \\
\hline
\end{tabular}

Table 3: Volumetric flow rate with mesh size (3D case).

\begin{tabular}{lll}
\hline \multicolumn{2}{c}{ Volumetric flow rate $\left[\mathrm{m}^{3} / \mathrm{s}\right]$} \\
\hline $1,688,208$ elements & $3,593,048$ elements & $3,821,304$ elements \\
$3.363 \mathrm{e}-3$ & $3.405 \mathrm{e}-3$ & $3.413 \mathrm{e}-3$ \\
\hline
\end{tabular}

\subsection{Exposure chamber}

Figure 5(a) presents the experimental setup used for the in vivo study. Rats will be exposed to $\mathrm{TiO}_{2} \mathrm{NPs}$. The exposure chamber (Unifab, Kalamazoo, USA) is $0.5 \mathrm{~m}^{3}$ and is equipped with two inlets located in the upper part and one outlet located in the lower part. In this section, the Eulerian approach, eqn (17), is applied to predict the transport, diffusion, and diffusional losses of airborne $\mathrm{TiO}_{2}$ particles in the exposure chamber. 


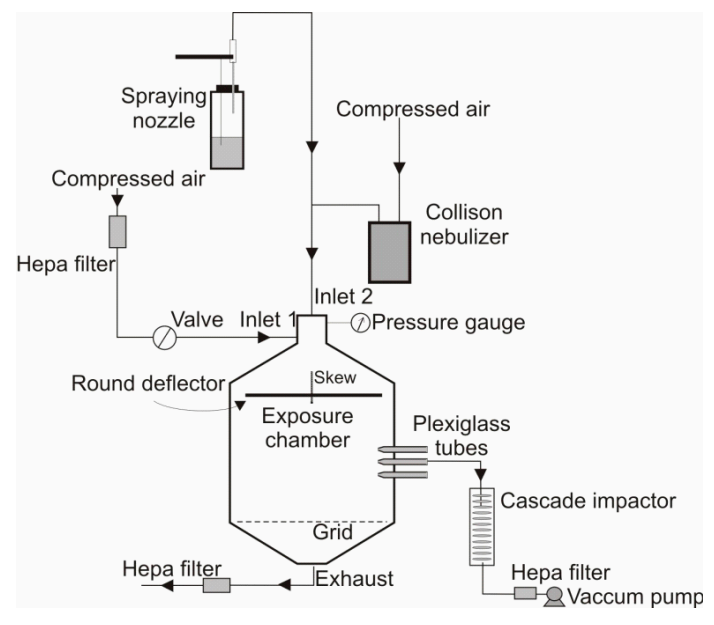

(a)

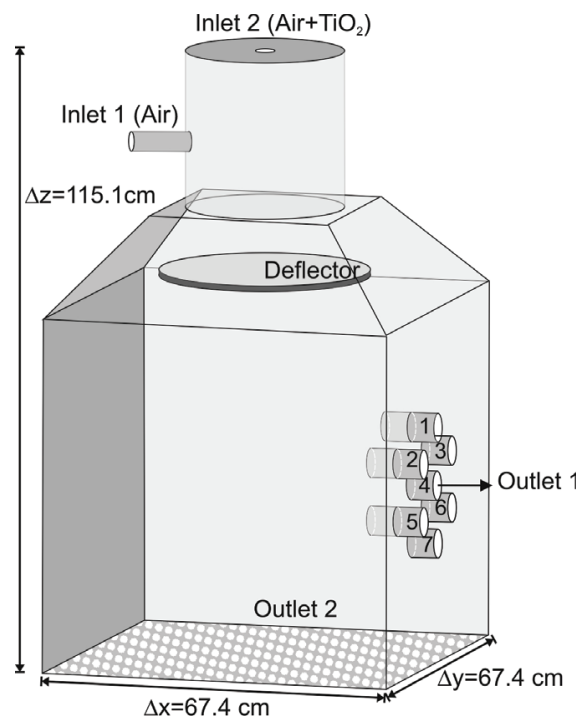

(b)

Figure 5: Schematic view of the experimental setup (a) and the computational domain (b).

As indicated in Fig. 5, airborne $\mathrm{TiO}_{2} \mathrm{NPs}$ are delivered into the chamber at inlet 2 by a 24-jet Collison nebulizer. The aerosol from inlet 2 is then mixed with and diluted by filtered fresh air admitted into the chamber via inlet 1. A round deflector plate in the upper part ensures thorough and even distribution of airflow. The inhalation chamber can be separated into two distinct zones. A mixing zone delimited by the top cylindrical part and the circular plate at the bottom and an exposure zone (or breathing zone) where the NPs mass fraction must be kept as uniform as possible with no stagnant areas. Measurements in the exposure zone show that the generated NP distribution can be approximated by a single mean aerodynamic diameter. The aerosol size distribution data obtained from a cascade impactor (Electrical Low Pressure Impactor, Dekati Inc.) located at inlet 2 presents a maximum concentration of airborne $\mathrm{TiO}_{2}$ on the order of $9.40 \times 10^{6}$ particles $/ \mathrm{cm}^{3}$, with a relatively narrow size distribution around a mean aerodynamic diameter of $26 \mathrm{~nm}$, as reported by Morency and Hallé [24].

A 3D view of the exposure chamber computational domain is presented in Fig. 5(b). The model width $(\Delta x)$, length $(\Delta y)$, and height $(\Delta z)$ are $67.4,67.4$, and $115.3 \mathrm{~cm}$, respectively. The diameter of each of the two inlets is $3.8 \mathrm{~cm}$. To reduce computational time, the bottom part of the exposure chamber was not considered in the numerical model. Therefore, the grid located at the bottom is modeled as an outlet vent. An outlet vent is an infinitely thin surface with a pressure drop proportional to the dynamic pressure of the flow. The velocity distributions at inlets 1 and 2 are considered to be uniform. No-slip conditions are imposed at walls for the velocity components and a mass concentration of 0 is imposed for the NPs. Since analyzing the flow inside the chamber is complex and computationally expensive, only one mesh density has been tested for this particular case. The unstructured mesh contains 1,912,973 elements and grid refinement near the walls guarantees a maximum $\mathrm{y}^{+}$value that is $<4$. 


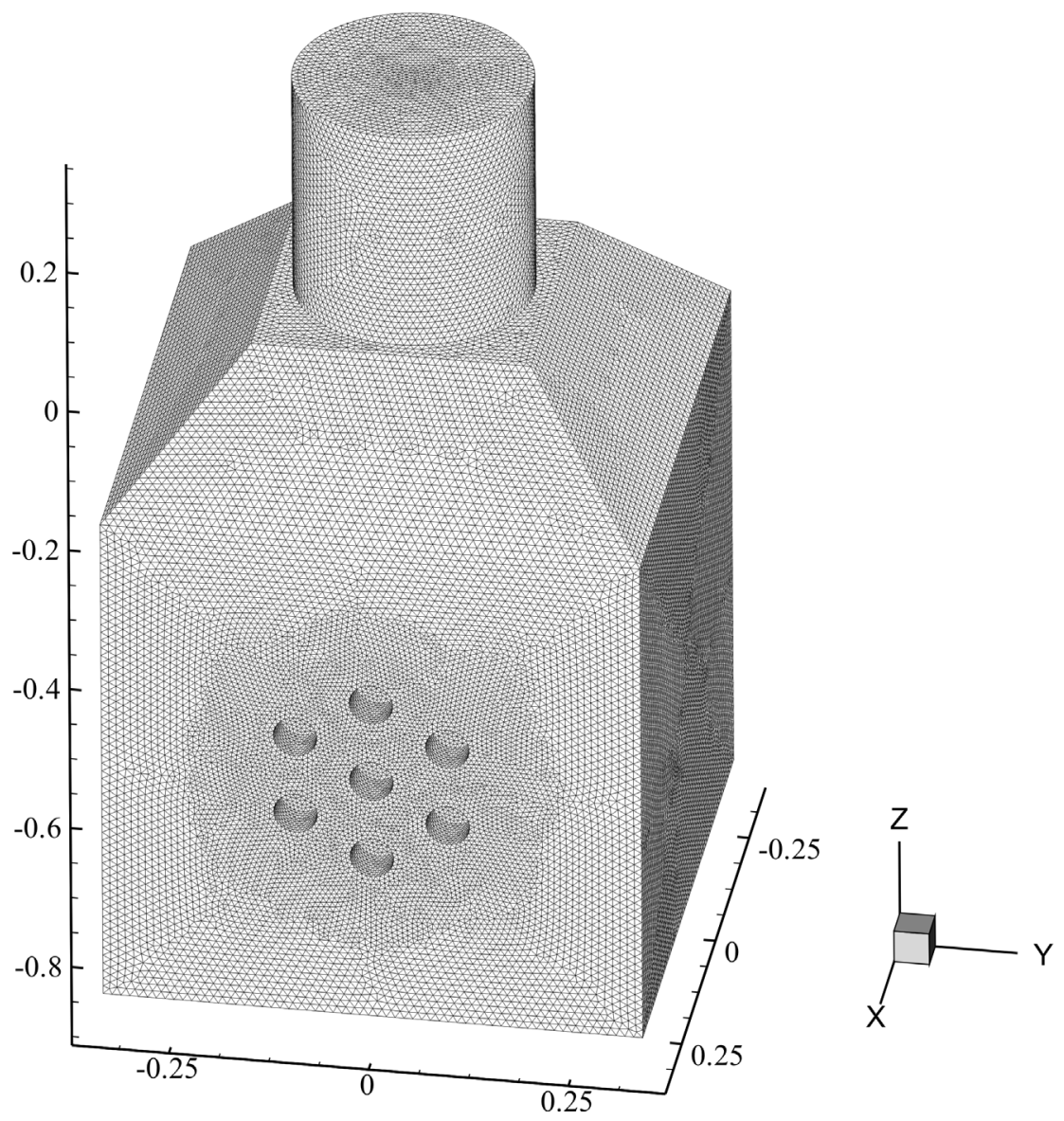

Figure 6: Unstructured surface mesh for the exposure chamber.

Figure 6 shows a representation of the unstructured surface mesh. This figure shows that the mesh density is slightly higher in the mixing region where both velocity and NP concentration gradients are higher. More grid nodes are also allocated in the Plexiglas tubes region. Note that in the nose-only inhalation exposure study, rats will be placed into Plexiglas restraint tubes with a hole at one end through which the animals can breathe.

A 2D slice of the iso-velocity contour of the mixing and breathing zone is presented in Fig. 7(a). The maximum air velocity from inlets 1 and 2 is $0.97 \mathrm{~m} / \mathrm{s}$. The air jets created from the supply vents collide in the mixing zone, creating a non-symmetrical distribution of the flow. The velocity then drop rapidly as the air- $\mathrm{TiO}_{2}$ mixture is driven to the lower part of the chamber. The maximum velocity drops to $0.5 \mathrm{~m} / \mathrm{s}$ at $\mathrm{z}=0.0 \mathrm{~m}$. Weak vortex areas are observed in the exposure zone. In this zone, the average velocity is around $30 \mathrm{~cm} / \mathrm{s}$. A central tube used for aerosol sampling created a suction zone with a significant velocity gradient, while the velocity near tubes 1 and 7 (Fig. 5(b)) remained close to zero.

Figure 7(b) presents the 2D mass fraction distribution of NPs in the mixing zone at $y=0.0 \mathrm{~m}$. We observed a significant variation of concentrations in this zone. The airborne NPs resulting 


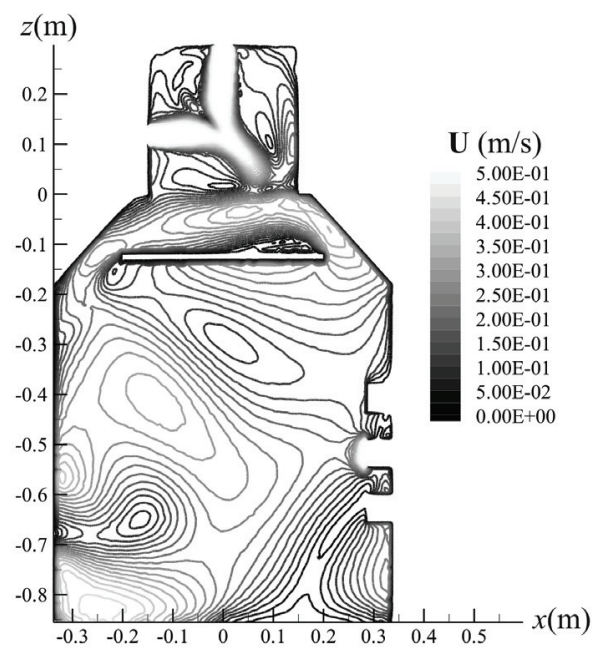

(a)

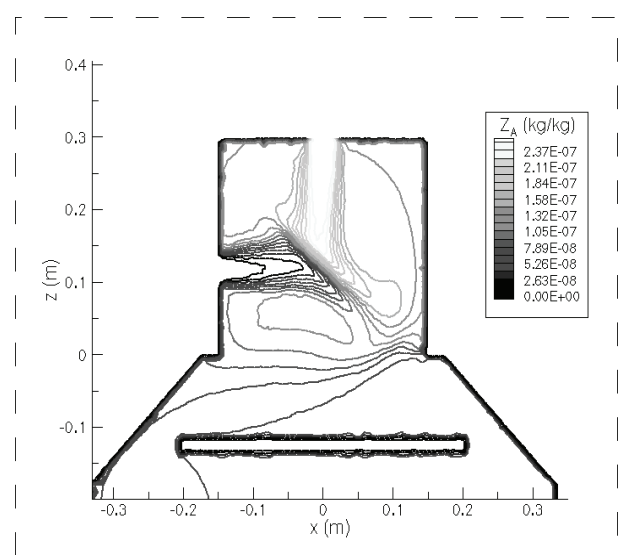

(b)

Figure 7: Velocity contours (a) and NP concentration in the mixing zone (b).

from inlet 2 at a mass concentration of $2.89 \times 10^{-7} \mathrm{~kg} / \mathrm{kg}$ is diluted by the fresh air coming from inlet 1 . The averaged mass concentration at $\mathrm{z}=0.0 \mathrm{~m}$ drops to $1 \times 10^{-7} \mathrm{~kg} / \mathrm{kg}$. Assuming a mean diameter of $26 \mathrm{~nm}$, this mass concentration corresponds to a number concentration of $3.25 \times 10^{6}$ particles $/ \mathrm{cm}^{3}$. Hinds [3] presents some data that can be used to estimate the time required to halve the number of particles by coagulation. For an initial concentration of $3 \times 10^{6}$ particles $/ \mathrm{cm}^{3}$, the time require to halve the number of particles by coagulation is on the order of $20 \mathrm{~min}$. The actual air exchange rate in the chamber is $17.2 \mathrm{~h}^{-1}$; at this level the $\mathrm{NP}$ residence time is estimated at $3.30 \mathrm{~min}$. Thus, it seems reasonable to neglect the coagulation of NPs as a first approximation.

Figure 8 shows the velocity streamlines. It is not possible to follow each individual streamline. The streamlines are uniformly dispersed in the exposure zone, which indicates that good mixing occurs in the chamber. There are several vortices oriented in various directions, but no clear flow pattern can be identified.

Table 4 presents the predicted mass fraction of NPs in the exposure chamber at seven location points. These seven points are located on the centerline of each of the seven Plexiglas tubes at $1 \mathrm{~cm}$ from the tubes' tip. For the simulated ventilation and aerosol generation conditions, the NP concentration in the rats breathing zone is fairly uniform. The averaged mass fraction is $6 \times 10^{-8} \mathrm{~kg} / \mathrm{kg}$ of air, with a standard deviation of $0.13 \times 10^{-8} \mathrm{~kg} / \mathrm{kg}$ of air. Table 4 also shows the measured mass fraction at the sampling tube (tube 4 ). Having access to a single cascade impactor, mass fraction measurement at the center tube gives us the most representative value at which rats will be exposed. The agreement between the experimental and predicted mass fraction is satisfactory, especially considering that (i) the exact location of the Dekati sampling system probe is not known precisely and (ii) phenomena, such as NPs, bouncing from the collection surface, the collection of particles other than those on the impaction plate, and the collection efficiency curve introduce uncertainty in the experimental measurements. 


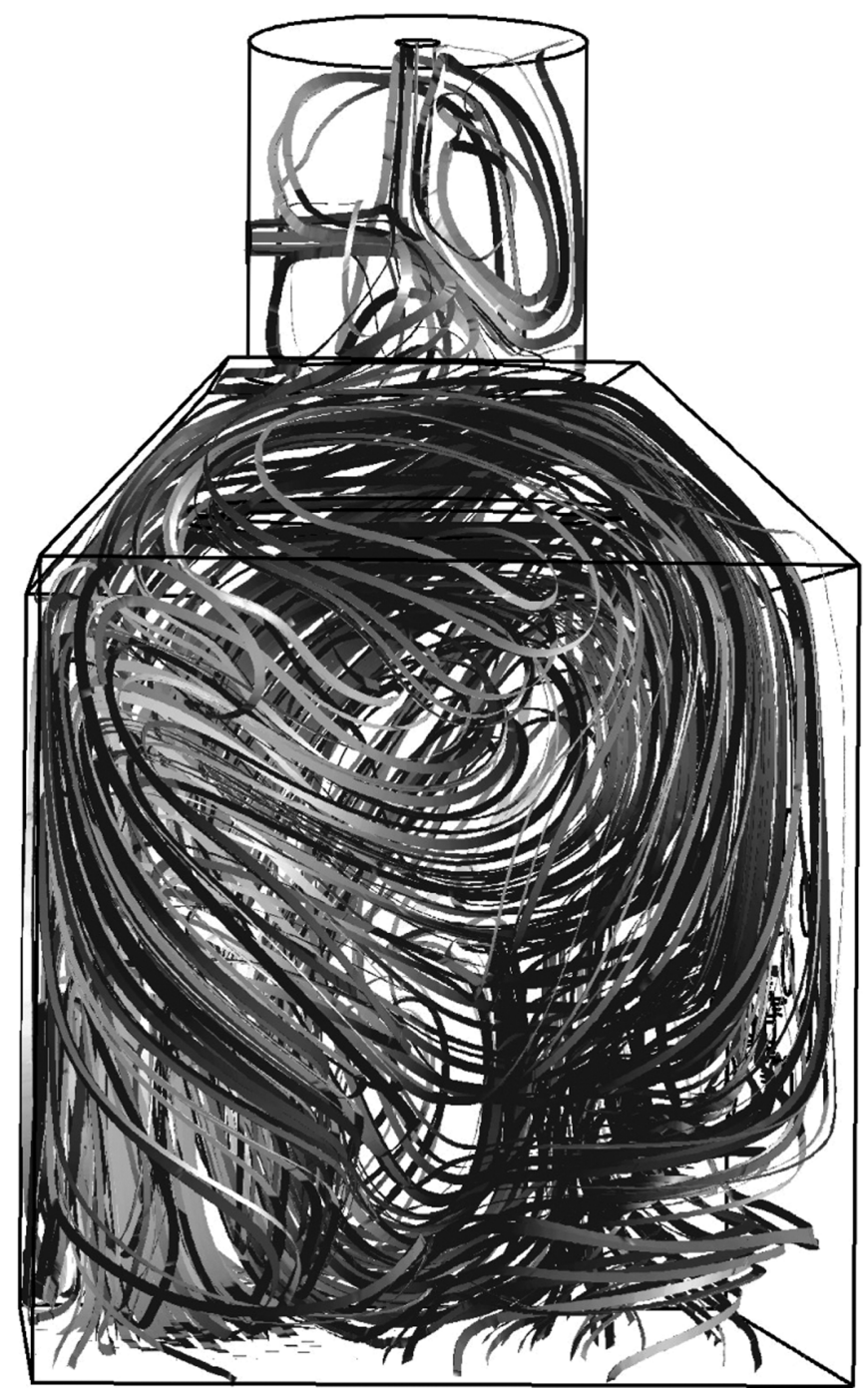

Figure 8: Velocity streamlines.

Table 4: Predicted and measured mass fraction.

\begin{tabular}{lrrrrrrr}
\hline Tube (Fig. 5(b)) & 1 & 2 & 3 & 4 & 5 & 6 & 7 \\
\hline $\mathrm{Z}_{\mathrm{A}}\left(\times 10^{-8} \mathrm{~kg} / \mathrm{kg}\right)$ (numerical) & 6.12 & 6.15 & 5.90 & $\begin{array}{l}6.02 \\
\text { (n) }\end{array}$ & 6.04 & 5.80 & 5.92 \\
$\mathrm{Z}_{\mathrm{A}}\left(\times 10^{-8} \mathrm{~kg} / \mathrm{kg}\right)$ (measured) & & & & 5.59 & & & \\
\hline
\end{tabular}

\section{CONCLUSION}

A simple model of NP transport, based on the assumption that NPs behave as a passive contaminant, is used to predict their concentration in an animal exposure chamber. The NPs are thus transported by convection and diffusion only. The validity of the approach is first verified by comparison against existing empirical correlations derived to estimate particle deposition inside a room. 
First, a 2D room was studied. For this $2 \mathrm{D}$ room, the numerical predictions agree well with recently derived correlations to predict decay coefficients, as long as NP size is $<100 \mathrm{~nm}$. The decay coefficient changes log-linearly with the particle diameter. The slope of the numerical results corresponds to a value of $n=2.94$ in the Crump and Seinfeld [11] twoparameter empirical correlation. This value is in agreement with Lai and Nazaroff [14].

A 3D room was studied next. This study indicated that the diffusive boundary layer is thinner than the momentum boundary layer, because of the low diffusion coefficient values. Particular attention must be paid to node spacing near walls to make sure that the diffusive boundary layer is captured correctly. For the 3D room, the numerical diffusion coefficient varies log-linearly with the diffusion coefficient, with a slope of 0.86 . This slope is between the value predicted by the Corner and Pendlebury correlation [10] and the value predicted by Crump and Seinfeld's [11] two-parameter empirical correlation, with a value of $n=2.9$.

Finally, predicted mass fraction obtained with the passive contaminant assumption compare well with the experimental result for a specific geometry of an animal exposure chamber. The upper part of the exposure chamber acts as a mixing chamber and uniformly disperses the NPs from the inlet, thus reducing NP concentration and preventing coagulation. The size of NPs is $<100 \mathrm{~nm}$ and thus gravity settling is negligible. Numerical predictions show that mass concentration is uniform in the seven Plexiglas tube tips. Although a single mesh density was tested, the predicted mass fraction of NPs at the sampling tube agrees quite well with experimental measurement. Thus, a single-point experimental measurement of the mass fraction appears to be sufficient for further inhalation study purposes.

\section{REFERENCES}

[1] Aitken, R.J., Creely, K.S. \& Tran, C.L., Nanoparticles: an occupational hygiene review. Health \& Safety Executive, p. 113, 2004.

[2] Ostiguy, C., Roberge, B., Menard, L. \& Endo, C.-A., Guide de bonnes pratiques favorisant la gestion des risques relies aux nanoparticules de synthese. IRSST, p. 63, 2008.

[3] Hinds, W.C., Aerosol Technology: Properties, Behavior, and Measurement of Airborne Particles. John Wiley \& Sons: New York, 1999.

[4] Friedlander, S.K., Smoke, Dust, and Haze - Fundamentals of Aerosol Dynamics. Oxford University Press: New York, 2000.

[5] Rudiak, V.Y., Krasnolutskii, S.L., Nasibulin, A.G. \& Kauppinen, E.I., Methods for measuring the diffusion coefficient and sizes of nanoparticles in a rarefied gas. Doklady Physics, 47(10), pp. 758-761, 2002. doi: http://dx.doi.org/10.1134/1.1519325

[6] Gussman, R.A., On the aerosol particle slip correction factor. Journal of Applied Meteorology, 8, pp. 999-1001, 1969. doi: http://dx.doi.org/10.1175/15200450(1969)008<0999:OTAPSC >2.0.CO;2

[7] Morency, F., Halle, S., Dufresne, L. \& Emond, C., Evaluation of diffusion models for airborne nanoparticles transport and dispersion. Advances in Fluid Mechanics VII. WIT Press: Ashurst, England, 2008.

[8] Maynard, A.D. \& Kuempel, E.D., Airborne nanostructured particles and occupational health. Journal of Nanoparticle. Research, 7, pp. 587-614, 2005. doi: http://dx.doi. org/10.1007/s11051-005-6770-9

[9] Lai, A.C.K., Particle deposition indoors: a review. Indoor Air, 12(4), pp. 211-214, 2002. doi: http://dx.doi.org/10.1046/j.0905-6947.2002.1r159a.x

[10] Corner, J. \& Pendlebury, E.D. The coagulation and deposition of a stirred aerosol. Proceedings of the Physics Society Section B, 64, pp. 645-654, 1951. doi: http://dx.doi. org/10.1088/0370-1301/64/8/304 
[11] Crump, J.G. \& Seinfeld, J.H., Turbulent deposition and gravitational sedimentation of an aerosol in a vessel of arbitrary shape. Journal of Aerosol Science, 12, pp. 405-415, 1981. doi: http://dx.doi.org/10.1016/0021-8502(81)90036-7

[12] Chen, B.T., Yeh, H.C. \& Cheng, Y.S., Evaluation of an environmental reaction chamber. Aerosol Science and Technology, 17(1), pp. 9-24, 1992. doi: http://dx.doi. org/10.1080/02786829208959556

[13] Benes, M. \& Holub, R.F., Aerosol wall deposition in enclosures investigated by means of a stagnant layer. Environment International, 22(Suppl 1), pp. S883-S889, 1996. doi: http://dx.doi.org/10.1016/S0160-4120(96)00197-3

[14] Lai, A.C.K. \& Nazaroff, W.W., Modeling indoor particle deposition from turbulent flow onto smooth surfaces. Journal of Aerosol Science, 31(4), pp. 463-476, 2000. doi: http:// dx.doi.org/10.1016/S0021-8502(99)00536-4

[15] Menter, F.R., Two-equation eddy-viscosity turbulence models for engineering applications. AIAA Journal, 32(8), pp. 1598-1605, 1994. doi: http://dx.doi.org/10.2514/3.12149

[16] Fluent Inc. Fluent 6.3 User's Guide, 2006.

[17] El Gharbi, N., Absi, R., Benzaoui, A. \& Bennacer, R., An improved near-wall treatment for turbulent channel flows. International Journal of Computational Fluid Dynamics, 25(1), pp. 41-46, 2011. doi: http://dx.doi.org/10.1080/10618562.2011.554832

[18] Lechner, M.D. \& Machtle, W., Determination of the particle size distribution of 5-100-nm nanoparticles with the analytical ultracentrifuge: consideration and correction of diffusion effects. Progress in Colloid and Polymer Science, 113, pp. 37-43, 1999. doi: http://dx.doi. org/10.1007/3-540-48703-4 6

[19] Hu, S.C., Chuah, Y.K. \& Yen, M.C., Design and evaluation of a mini-environment for semi-conductor manufacture processes. Building and Environment, 37, pp. 201-208, 2002. doi: http://dx.doi.org/10.1016/S0360-1323(00)00095-0

[20] Zhao, B. \& Wu, J. Numerical Investigation of particles diffusion in a clean room. Indoor and Built Environment, 14(6), pp. 459-479, 2005. doi: http://dx.doi. org/10.1177/1420326X05060190

[21] Zhang, Z., Kleinstreuer, C. \& Kim, C.S., Airflow and nanoparticle deposition in a 16-generation tracheobronchial airway model. Annals of Biomedical Engineering, 36(12), pp. 2095-2110, 2008. doi: http://dx.doi.org/10.1007/s10439-008-9583-z

[22] Kumar, P., Garmory, A., Ketzel, M., Berkowicz, R. \& Britter, R., Comparative study of measured and modelled number concentrations of nanoparticules in an urban street canyon. Atmospheric Environment, 43, pp. 949-958, 2009. doi: http://dx.doi.org/10.1016/j. atmosenv.2008.10.025

[23] Lai, A.C.K. \& Chen, F.Z., Comparison of a new Eulerian model with a modified Lagrangian approach for particle distribution and deposition indoors. Atmospheric Environment, 41, pp. 5249-5256, 2007. doi: http://dx.doi.org/10.1016/j.atmosenv.2006.05.088

[24] Morency, F. \& Halle, S., Modelling nanoparticle transport in an animal exposure chamber: a comparison between numerical and experimental measurements. Advances in Fluid Mechanics VIII. WIT Press: Algarve, Portugal, 2010.

[25] Dzhaugashtin, K.E., The critical jet flow regime. Fluid Dynamics, 25(3), pp. 335-339, 1990. doi: http://dx.doi.org/10.1007/BF01049812

[26] Crump, J.G., Flagan, R.C. \& Seinfeld, J.H., Particle wall loss rates in vessels. Aerosol Science and Technology, 2(3), pp. 303-309, 1983. doi: http://dx.doi. org/10.1080/02786828308958636 